\title{
ON SINGULARITIES OF CAPILLARY SURFACES IN THE ABSENCE OF GRAVITY
}

\author{
V. ROYTBURD \\ Lawrence Berkeley Laboratory \\ University of California \\ Berkeley, California 94720 \\ (Received June 16, 1982)
}

ABSTRACT. We study numerical solutions to the equation of capillary surfaces in trapezoidal domains in the absence of gravity when the boundary contact angle declines from $90^{\circ}$ to some critical value. We also discuss a result on the behavior of solutions in more general domains that confirms numerical calculations. KEY WORDS AND PHRASES. Capillarity, constant mean cuevature, critical contact angles.

1980 MATHEMATICS SUBJECT CLASSIFICATION CODE. 35220; 65N30.

\section{INTRODUCTION.}

From a mathematical point of view, the physical properties of a liquid in a capillary tube in the absence of gravity can be described with the help of only one parameter--the contact angle. This is the angle between the capillary surface and the walls of the cylinder. For walls of a homogeneous material, the contact angle must be constant along the boundary.

Concus and Finn [1] have shown that the capillary surface does not exist for all physically reasonable contact angle. They obtained a necessary condition for the existence of a solution. The condition gives an estimate of the contact angle in terms of the geometry of the base domain of the cylinder. In the case that gravity is absent, the estimate is essentially nonlocal, as it cannot be expressed by means of local geometrical characteristics of the boundary.

Finn in [2] noticed that, even for a polygonal domain, one cannot infer informaition on the existence of a solution from knowledge of the vertex angles alone. 
Employing the mentioned necessary condition, he has shown that, for any $\gamma_{0}$, $0<\gamma_{0}<$ $\pi / 2$, there exists a small deformation of a rectangular domain to a trapezoidal one such that there is no solution in the trapezoldal domain for contact angles less than $\gamma_{0}$. It should be mentioned that, for a rectangular domain, a solution exists for any angle larger than $\pi / 4$ and that, for the contact angle equal to $\pi / 2$, a (flat) capillary surface exists over any domain.

For contact angles between $\gamma_{0}$ and $\pi / 2$, the necessary condition, violated for $\gamma<\gamma_{0}$, is satisfied, but as far as we know there is no proof of existence of a regular capillary surface for this case. Nevertheless, one can calculate a solution numerically.

We describe the results of two kinds of numerical experiments. First, for a fixed trapezoid, we consider solutions as the contact angle declines from $\pi / 2$ to some apparently critical angle; and second, we consider the behavior of solutions as the base domain is deformed from a rectangle to some critical trapezoid (with the contact angle fixed). In both cases, the solutions have a clear tendency to verticality as criticality is approached.

In the last section, we give some explanation to these "experimental" facts. We show that for arbitrary domains, in the assumption of the existence of a regular capillary surface, the gradient of the solution tends to infinity over some characteristic curve when the contact angle tends to the critical value.

\section{CAPILLARITY PHENOMENA.}

We include here some information on capillarity phenomena. For a detailed presentation we refer to the article of Finn [3].

We consider a liquid partly filling a vertical cylinder with a base domain $\Omega$; the boundary of $\Omega$ is denoted by $\Sigma$. We assume that the height of the equilibrium free surface of the liquid in the cylinder is a single-valued smooth function $u(x, y)$. We assume also that the volume of the liquid is sufficiently large to cover the base of the cylinder entirely.

In the absence of gravity, the height $u(x, y)$ of the liquid oyer the bottom of the cylinder satisfies the equation 


$$
\begin{gathered}
\operatorname{div}\left(\frac{1}{W} \nabla u\right)=2 \mathrm{H} \\
\text { where } \nabla \mathrm{u}=(\partial \mathrm{u} / \partial \mathrm{x}, \partial \mathrm{u} / \partial \mathrm{y}), \mathrm{W}=\left(1+|\nabla \mathrm{u}|^{2}\right)^{1 / 2} .
\end{gathered}
$$

The constant $\mathrm{H}$ is the mean curvature of the liquid surface. It is defined by the cross-sectional shape of the cylinder and by the boundary condition satisfied by the free surface of the liquid at the cylinder wall.

We will demand the free surface make a prescribed contact angle $\gamma$ with the cylinder wall. Thus, the boundary condition is

$$
\frac{1}{W} \frac{\partial u}{\partial n}=\cos \gamma \text { on } \Sigma \text {. }
$$

Here, $\partial u / \partial n$ is the derivative with respect to the outward directed normal at the wall. Applying the divergence theorem to (2.1) with boundary condition (2.2), we obtain the relation between the mean curvature and the contact angle:

$$
2 \mathrm{H} \Omega=\sum \cos \gamma
$$

Here and also later we use the same symbols to denote domains and their measures. Eq. (2.3) is a necessary condition for the existence of the solution to (2.1) and (2.2). Concus and Finn [1] obtained, in addition, a more general condition than (2.3). Namely, let the cross-section $\Omega$ of the cylinder be cut into two parts, $\Omega *$ and $\Omega-\Omega$ * by a curve $\Gamma$, which intersects the boundary $\sum$ at points $\mathrm{p}_{1}$ and $\mathrm{p}_{2}$. Let $\Sigma *$ denote the part of $\Sigma$ cut off by $\Gamma$ and adjacent to $\Omega^{*}$. Then, applying the divergence theorem to the domain $\Omega *$ and taking into account that $|\nabla u / W| \leq 1$ along $\Gamma$ (as everywhere), one can obtain the estimate

$$
\cos \gamma \leq \mathrm{V}
$$

where

$$
\mathrm{V}=\min \frac{\Gamma / \Sigma}{|\Sigma * / \Sigma-\Omega * / \Omega|}
$$

The minimization is carried over all curves $\Gamma$ described above such that $\Sigma * / \Sigma-\Omega * / \Omega$ is nonzero. The estimate (2.4) holds if the solution to (2.1) and (2.2) exists.

3. COMPUTATIONAL EXPERIMENTS.

Now we consider equations (2.1) and (2.2) when the base domain is a trapezoid. For numerical experiments, an equilateral trapezoid was chosen with the following parameters: the long base $b=2 .$, the short base $a=1.3$, and the height $h=25$. The angles of the trapezoid are very close to $90^{\circ}$, approximately $90^{\circ} \pm 0.8^{\circ}$. This 
trapezoid has a sufficiently large altitude to exhibit clearly the nonexistence phenomenon under study, but not so large as to require an excessive number of mesh points for representation of the solution. The departure from a rectangle is sufficient to permit visualization of the change to the trapezoid when the shorter base is decreased from 2 to 1.3 .

For the trapezoid, the quantity $\mathrm{V}$ in $(2.4)$ giving an upper bound for the cosine of the contact angle can be calculated numerically. The minimum of the expression (2.5) for $\mathrm{V}$ is realized when $\Gamma$ is a particular circular arc connecting sides of the trapezoid. The calculation for the critical angle $\gamma_{0}$ for the trapezold with the above parameters gave an approximate value

$$
r_{0} \sim 57.6^{\circ}
$$

Since (2.1) and the boundary condition (2.2) are invariant under reflection with respect to the symmetry axis of the trapezoid, it is easy to show that the solution itself should be symmetric. Thus we may solye (2.1) in the half-trapezoid

$$
\mathrm{T}=\{(\mathrm{x}, \mathrm{y}) \mid 0 \leq \mathrm{y} \leq 25,0 \leq \mathrm{x} \leq 1-0.014 \mathrm{y}\}
$$

with the boundary condition (2.2) on the half-perimeter of the trapezoid and the boundary condition

$$
\left.\frac{\partial u}{\partial n}\right|_{x=0}=0
$$

The problen, was solved numerically using a suitable modified version of Brown's finite-element program [4] for the capillary problem in a rectangle. The domain was discretized employing a trapezoidal grid constructed as follows. Each base of the half trapezoid $T$ was divided equidistantly into $M$ parts and the height was divided equidistantly into $\mathrm{N}$ parts. Mesh points were formed by intersections of the mesh lines connecting the obtained points on the bases and the mesh lines parallel to the x-axis through the points of partition of the height.

The program used reduced quadratic polynomials as the basis functions for the finite-element method (the reduced quadratic element differs from the full biquadratic element by the elimination of the $x^{2} y^{2}$ term and omission of the centroid node). The resulting nonlinear algebraic problem is solyed using Newton's method.

Computations were carried out with $N=50, N_{1}=4$, and wer̨e repeated fọ $N=75, M=7$. 
The two series of computations showed very good consistency between their results. The method was tested for the $2 \times 25$ rectangle for contact angles close to $45^{\circ}$. The method converged for $\gamma \geq 46^{\circ}$ and did not converge for $\gamma \leq 45^{\circ}$ (the step of changing the contact angle in test calculations was $1^{\circ}$ ).

The results are depicted in figures 1-3, normalized by the addition of a constant so that $u(0,0)=0$. In figure 1 the surface height $u(0, y)$ along the symetry line is shown for several contact angles $\gamma$. The behavior of the solution along other mesh lines in the "y direction" differs very little from that of $u(0, y)$.

In figure 2, the variation of the surface height with $x$ is depicted for several values of $y$ for the case $\gamma=58^{\circ}$. Note that the optimal curve $\Gamma_{0}$, which gives the value $57.6^{\circ}$ for the contact angle, is a circular arc of radius 1.444 intersecting the symmetry line $x=0$ of the trapezoid at $y \sim 17.6$ and the slant edge at $y \sim 17.4$.

In figure 3 are depicted the surface heights $u(0, y)$ for a sequence of trapezoids ranging from the rectangle $(a=2)$ to the almost-critical one $(a=1.3)$ for $\gamma=58^{\circ}$. The tendency toward verticality is noticeable as criticality is approached. 4. ON THE GRADIENT OF THE SOLUTION.

Figure 1 indicates that, for $\gamma$ close to the critical angle, the graphs have inflection points where the derivative $u_{y}$ is maximal. The maximum of $u_{y}$ appears to occur in a small neiphborhood of the curve $\Gamma_{0}$ for which the minimum in the estimate (2.5) is realized. This property is not incidental. Actually, $\Gamma_{0}$ is the curve along which a discontinuity of the solution may arise. Namely, $\left.\frac{\partial u}{\partial n}\right|_{\Gamma_{0}} \rightarrow \infty$ as $\gamma \rightarrow \gamma_{0}$, where $\frac{\partial}{\partial n}$ is differentiation with respect to the outward normal to $\Gamma_{0}{ }^{\cdot}$ More precisely, the following is valid.

Theorem: Let a solution to equations (2.1) and (2.2) exist for $\gamma>\gamma_{0}$, where $\gamma_{0}$ is defined by the equality

$$
\cos \gamma_{0}=\min \frac{\Omega \Gamma}{\Gamma|\Sigma \star \Omega-\Omega \star \Sigma|}
$$

and let $\Gamma_{0}$ realize the minimum in $(2.6)$. Then

as $\gamma \rightarrow \gamma_{0}$

$$
\left|\int_{\Gamma_{0}} \frac{1}{\mathrm{~W}} \nabla \mathbf{u} \cdot \mathrm{n} \mathrm{ds}\right| \rightarrow \Gamma_{0}
$$

Proof: Let $\delta>0$ be small and 


$$
\cos \gamma_{0}>\cos \gamma>\cos \gamma_{0}-\delta
$$

We integrate both sides of (2.1) over the domain $\Omega *$ defined by $\Gamma_{0}$ (for the definition of $\Omega *$ see the paragraph preceding (2.4)). Applying the divergence theorem and expressing $\mathrm{H}$ through other parameters with the help of (2.2), we have

$$
\Sigma * \cos \gamma+\int_{\Gamma_{0} \mathrm{~W}} \stackrel{1}{\nabla} \nabla \cdot \mathrm{n} \quad \mathrm{ds}=\Omega * \frac{\Sigma \cos \gamma}{\Omega}
$$

Let $\Omega \star \Sigma-\sum \star \Omega>0$ (otherwise, we can obtain the necessary estimate for $\left.-\int_{\Gamma_{0}} W^{-1} \nabla \mathbf{u} \cdot \mathrm{n} \mathrm{ds}\right)$, then it follows that

$$
\begin{array}{cc}
\int_{\Gamma} \frac{1}{\mathrm{~W}} \nabla \mathrm{u} \cdot \mathrm{n} \quad \mathrm{ds}=\left\{\Omega * \Sigma / \Omega-\Sigma^{*}\right) \cos \gamma>\left(\Omega * \Sigma / \Omega-\Sigma^{*}\right)\left(\Gamma_{0} \Omega /(\Sigma \Omega *-\Sigma * \Omega)-\delta\right\} \\
=\Gamma_{0}-\delta(\Omega * \Sigma / \Omega-\Sigma *) \rightarrow \Gamma_{0} & \text { as } \delta \rightarrow 0 .
\end{array}
$$

The theorem is in a sense, an inverse to Concus and Finn's estimate of the critical angle (2.5).

By usual means, there can be derived from the theorem (by assumption of the regularity of the solution) the property that $\partial u / \partial n$ goes to infinity on $\Gamma_{0}$ as $\gamma \rightarrow \gamma_{0}$.

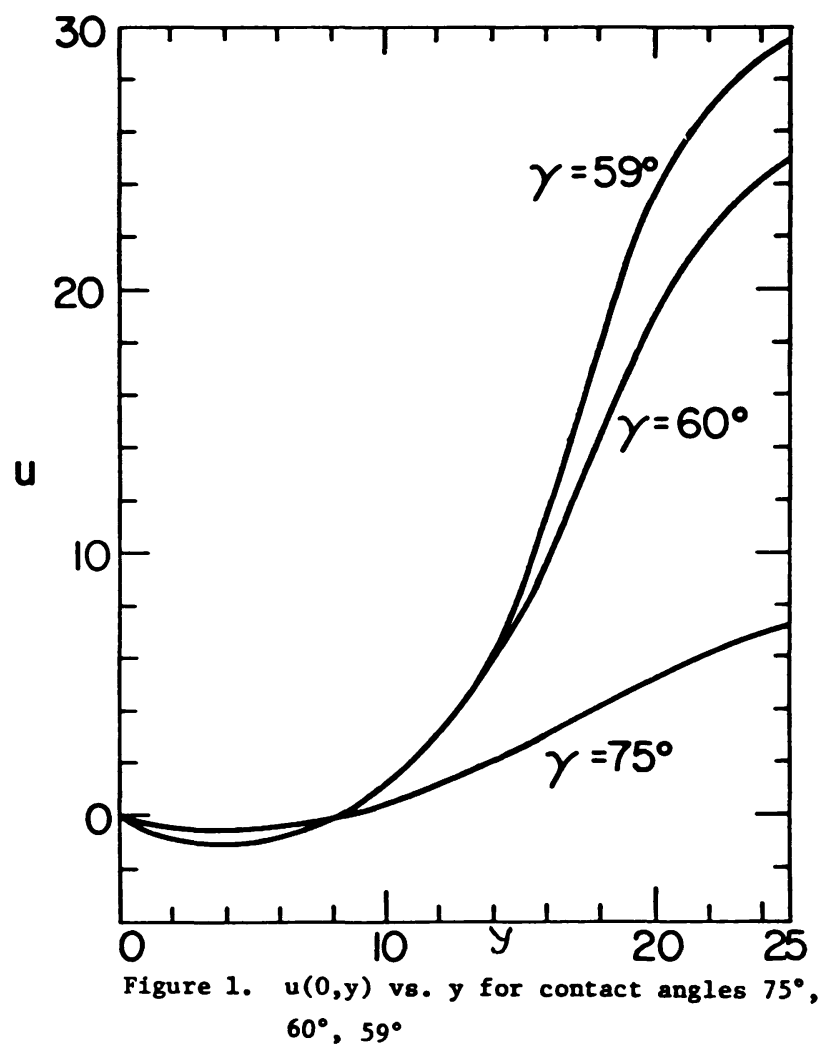




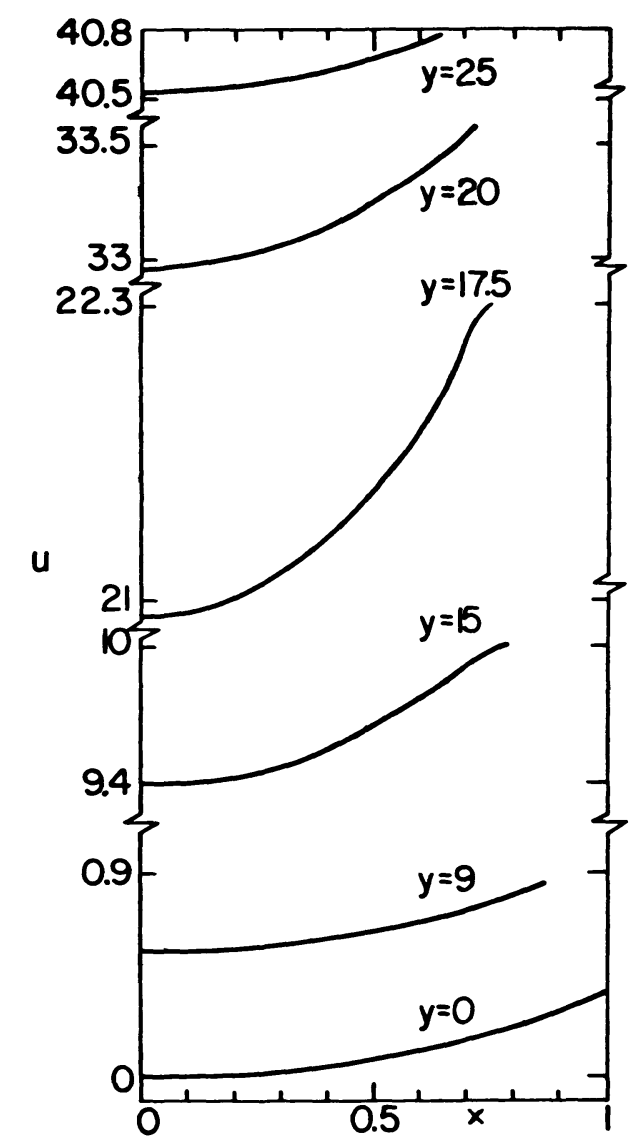

Figure 2. $u(x, y)$ vs. $x$ for $y=0,9$ $15,17.5,20,25 ; \gamma=58^{\circ}$

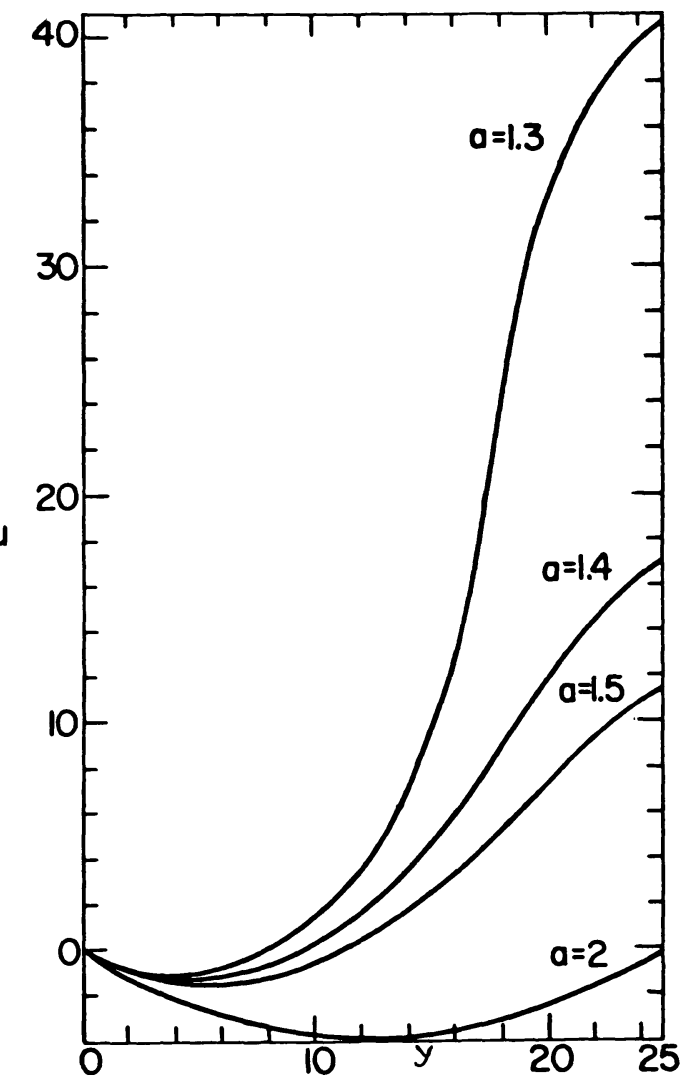

Figure 3. $u(0, y)$ vs. $y$ for $a=2,1.5,1.4$, $1.3 ; \gamma=58^{\circ}$

ACKNOWLEDGEMENTS. This work was carried out at the suggestion of Dr. Paul Concus. The author would like to thank Dr. P. Concus for his support and helpful discussions and Prof. J. B. Keller for comments. D. Culler assisted with calculations of the extremal circular arcs. This work was supported by NASA Lewis Laboratory under contract C-32819-D and in part by the Engineering, Mathematical, and Geosciences Division of the U.S. Department of Energy under contract W-7405-ENG-48.

\section{REFERENCES}

1. CONCUS, P. and FINN, R. On Capillary Free Surfaces in the Absence of Gravity, Acta Math. 132 (1974), 177-198.

2. FINN, R. Existence and Non-existence of Capillary surfaces, Manuscripta Math. 28 (1979), 1-11.

3. FINN, R. Capillarity Phenomena. Russian Math. Surveys 29:4 (1974), 131-152.

4. BROWN, R. A. Finite Element Methods for the Calculation of Capillary Surfaces, J. Comp. Phys. 33 (1979), 217-235. 


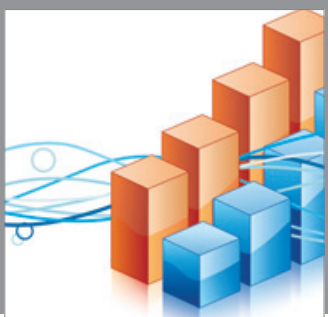

Advances in

Operations Research

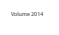

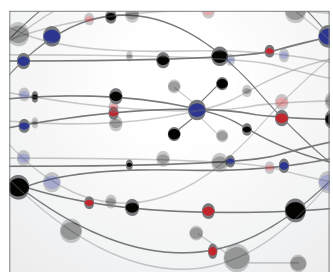

\section{The Scientific} World Journal
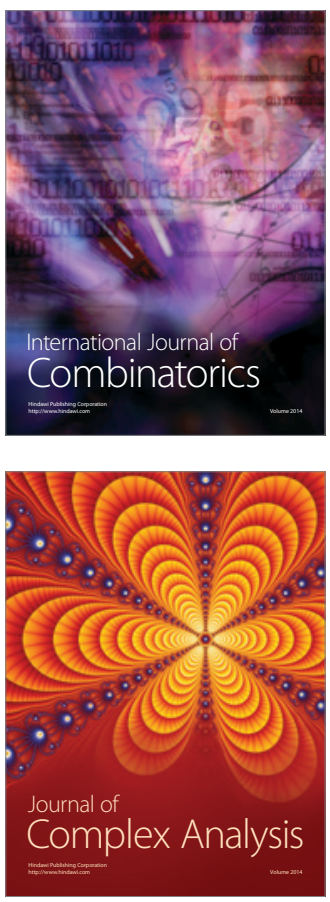

International Journal of

Mathematics and

Mathematical

Sciences
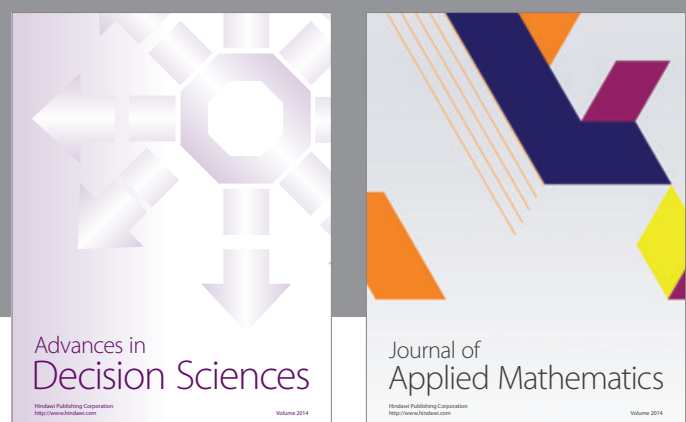

Journal of

Applied Mathematics
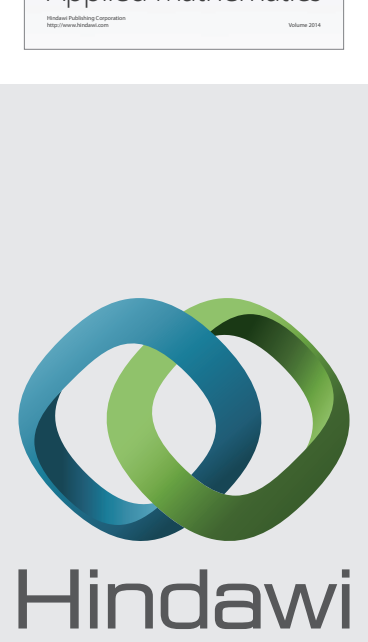

Submit your manuscripts at http://www.hindawi.com
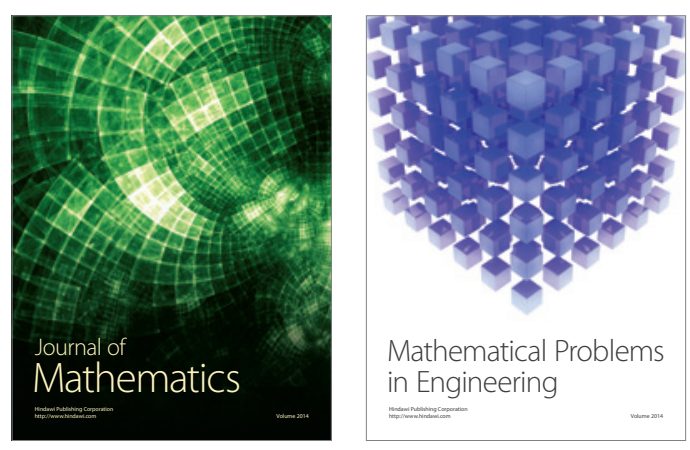

Mathematical Problems in Engineering
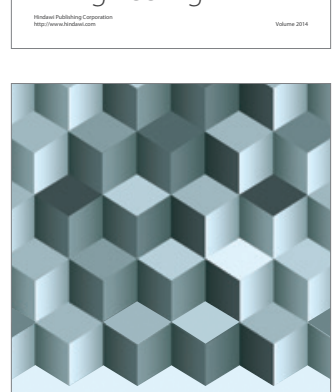

Journal of

Function Spaces
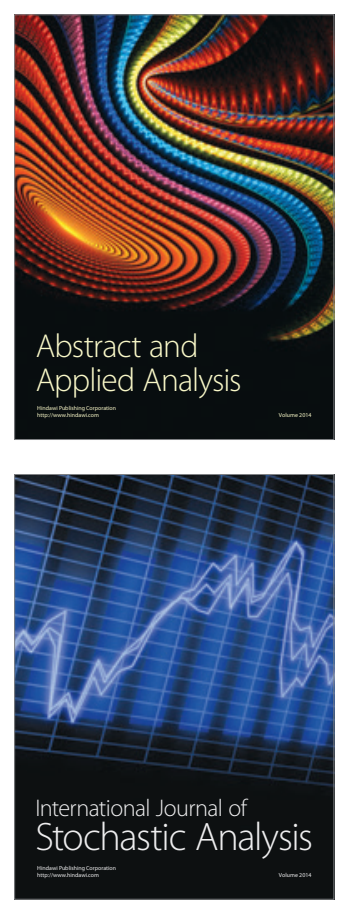

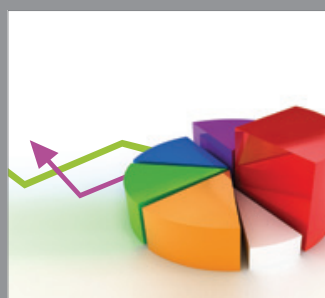

ournal of

Probability and Statistics

Promensencen
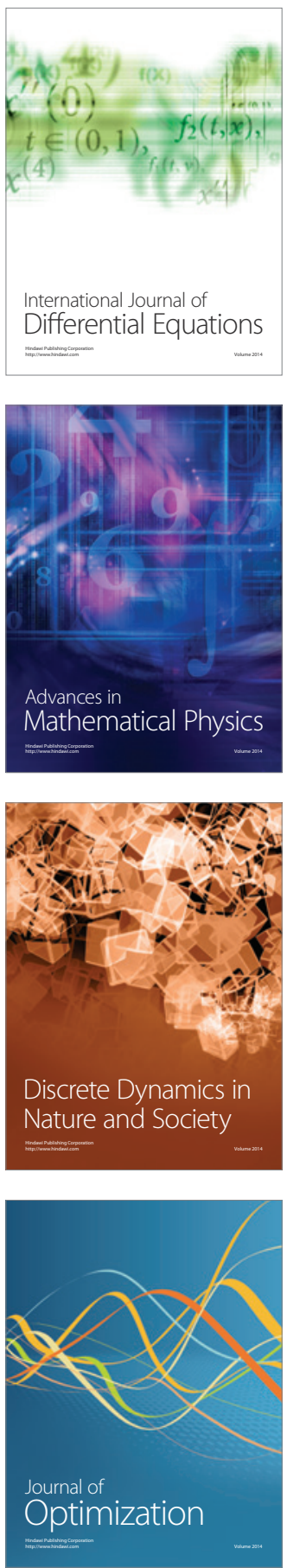